\title{
LE CONTRÔLE SOCIAL DU MONDE DES AFFAIRES : UNE ÉTUDE INSTITUTIONNELLE
}

\author{
Ana Maria FALCONI, Karima GUENFOUD, \\ Emmanuel LAZEGA, Claire LEMERCIER \\ et Lise MOUNIER ${ }^{1}$
}

Partage des coûts du contrôle et régulation conjointe des marchés

Une forme importante d' " encastrement " (Granovetter, 1985) des transactions économiques dans un contexte social est constituée

1. La recherche dont cet article est extrait a été financée par le GIP Mission de recherche " Droit et justice " du ministère de la Justice. Cette étude a été menée dans le cadre des activités du CLERSÉ-CNRS (Lille) et du LASMAS-CNRS (Paris). Nous remer-cions les juges du Tribunal de commerce de Paris de leur accueil, la Mission de recherche "Droit et justice " de son soutien, ainsi que Philippe Rollet, doyen de la faculté des Sciences économiques et sociales de l'Université de Lille I, Chantal Figueredo et Sylvie Kleczewski de leur aide pour la gestion du programme de recherche dont ce texte est issu. Enfin nous remercions Véronika Nagy, Elsa Ramos et Stéphanie Ver-meersch de leur participation à cette étude. 
par ce que Buskens et al. (2003) appellent «encastrement institutionnel $»^{2}$. Les règles du jeu fournies par les institutions donnent aux acteurs qui acceptent ces contraintes la possibilité de réduire les risques liés aux transactions marchandes (North, 1990; Schelling, 1960 ; Williamson, 1985, 1996). Les entreprises se surveillent mutuellement et apprennent à s'adapter aux contextes institutionnels et aux organisations de régulation de toutes sortes sanctionnant les écarts aux règles ainsi établies (Abulafia, 1997) ${ }^{3}$. La sociologie économique s'est, depuis ses débuts, intéressée à la création, au fonctionnement et à l'évolution des institutions contrôlant le fonctionnement des marchés. S'appuyant sur les théories weberiennes ${ }^{4}$ concernant les "organisations de régulation de l'économie" (wirtschaftsregulierender Verbände) comme les banques, les bourses ou les institutions étatiques, Hamilton et Feenstra (1998) ou Whitley (1992) ont montré, par exemple, que différentes conditions institutionnelles initiales ${ }^{5}$ avaient des effets spécifiques sur l'émergence de formes de capitalisme différentes.

L'État a traditionnellement fourni ces institutions, permettant notamment au monde des affaires de gérer les risques et les pro-

2. Voir aussi à ce sujet Macaulay (1986) et Raub et Weesie (2000).

3. Les économistes comme Williamson (1975) ou North et Thomas (1976) pensent les institutions sociales (par opposition aux systèmes de prix), y compris les institutions juridiques, comme des solutions aux inefficacités des marchés, inefficacités liées aux coûts de transactions et aux risques pris par les entrepreneurs. Les activités marchandes ont besoin de règles, par exemple de règles de droit formelles. Parmi ces dernières, le droit de propriété est un exemple classique (Campbell et Lindberg, 1991; North, 1990). Ces structures institutionnelles procurent la stabilité dont ont besoin les entrepreneurs (Fligstein, 2001). Elles imposent donc des règles du jeu qui, en structurant les incitations et par conséquent les performances des économies, atténuent les risques liés aux activités de production et d'échange marchand. Elles créent un contexte où les engagements, contractuels ou non, deviennent crédibles, en particulier là où les échanges marchands ont tendance à devenir impersonnels (Buskens et al., 2003). Dans cette mouvance dite "néoinstitutionnaliste" en économie, des historiens comme Greif (1998) ont montré l'importance du développement d'institutions formelles et impersonnelles pour l'émergence de formes durables de capitalisme. D'autres courants, comme celui de la law \& economics, font des institutions des mécanismes de réduction des coûts de mise en œuvre du droit. Cependant, il leur est difficile, dans le cadre théorique qu'elles se sont fixé, de tirer toutes les leçons de l'incomplétude des contrats en ce qui concerne les obligations des parties. Voir à ce sujet, par exemple, Posner (2000), en particulier le chapitre 9 ( "Contract law and commercial behavior" ").

4. Voir aussi Swedberg $(1998,99-107)$ sur les pré-requis juridiques de l'émergence du capitalisme selon Weber.

5. Les théories économiques dites "évolutionnistes" (evolutionary) de Nelson et Winter (1982) et leur concept de dépendance de sentier (path dependency) décrivent bien l'importance des conditions initiales pour les trajectoires des systèmes économiques et font écho en ce sens aux théories weberiennes. D'autres (Evans, Rueschmeyer et Skocpol, 1985 ; Evans, 1995) affirment que c'est l'État (et non les marchés) qui a été le principal acteur du développement des différentes formes de capitalisme connues. 
blèmes que posent la concurrence et l'activité contractuelle. Mais le monde des affaires a participé à la construction de ces institutions, tout comme à l'équipement juridique des marchés. En effet, les arrangements institutionnels régulant les marchés varient selon qu'ils pèsent ex ante sur l'activité contractuelle ou ex post sur la résolution des conflits résultant de cette activité. La résolution des conflits par des tierces parties peut passer par les tribunaux, mais aussi par la médiation privée, l'arbitrage, ou encore des systèmes plus complexes comme celui que nous nous proposons d'examiner ici : les juridictions consulaires comme le tribunal de commerce français.

Le monde des affaires a très tôt participé au partage des coûts du contrôle social de ses activités économiques, c'est-à-dire à ce que nous avons appelé, à la suite de Jean-Daniel Reynaud (1989), une forme de régulation "conjointe " du monde des affaires (Lazega et Mounier, 2002, 2003 a) ${ }^{6}$. En Europe, c'est le monde des affaires qui a d'abord créé ses propres régulations. Mais il a très tôt recouru à l'État, dans un rapport complexe, pour obtenir de meilleurs moyens d'officialisation et de sanction de ses normes. Au cours des $\mathrm{XI}^{\mathrm{e}}$ et $\mathrm{XII}^{\mathrm{e}}$ siècles, d'abord dans les cités-États qu'ils dominaient, les commerçants européens développèrent leur propre droit commercial, qui en vint à coexister avec d'autres droits, comme le droit canonique. L'achat, la vente, le transport, l'assurance étaient régulés par un droit issu des corporations marchandes, que l'on a pu appeler, en raccourci, la lex mercatoria européenne (Berman, 1983), car, malgré des variantes locales, de grands principes se retrouvaient de place de commerce en place de commerce. On a ainsi pu dire que la lex mercatoria du Moyen Âge a été le fondement juridique du capitalisme (Weber, 1894). Les marchands, qui constituaient un groupe social relativement distinct des autres et aux intérêts économiques bien spécifiés, avaient leurs propres tribunaux et élisaient des juges parmi leurs pairs (comme les artisans le faisaient dans les guildes, métier par métier, mais dans un regroupement plus large de tous les types de commerce sur une base locale). On y délivrait une justice rapide sans argumentation juridique sophistiquée, fondée en équité, sans avocats professionnels. Les commerçants contrôlaient ce qui se passait dans leurs marchés et foires, mais parallèlement, ils faisaient souvent appel au pouvoir d'État pour mettre en œuvre les décisions qu'ils prenaient - ce qui ne signifie pas qu'ils ne pouvaient pas, dans certaines cir-

6. Voir aussi à ce sujet Ayres et Braithwaite (1992), Black (1984), Dunworth et Rogers (1996), Reiss (1984), Stone (1975) et Vaughan (1983). 
constances, compter seulement sur d'autres moyens de contrainte comme la réputation (Milgrom, North et Weingast, 1990).

Cette lex mercatoria évolua ainsi différemment selon les pays, en fonction de leurs types d'État (plus ou moins absolutiste) et de droit (entre common law et codification). En France, en particulier, c'est une forme spécifique d'institution qui en émergea à partir du XVI siècle : les tribunaux de commerce. Ceux-ci apparaissent comme un compromis visant à préserver les avantages de deux formes de régulation, endogène et exogène : tout en conservant les principes de la lex mercatoria (juges commerçants, justice rapide et peu chère, respect des usages du commerce), ils sont intégrés dans l'administration judiciaire (bénéficiant de ses moyens de sanction) et appliquent très tôt (dès la fin du XVII ${ }^{e}$ siècle) des lois commerciales générales. Ce compromis tient depuis le $\mathrm{XVI}^{\mathrm{e}}$ siècle, malgré des critiques régulières de son caractère hybride entre institution marchande et étatique (critiques portant sur les risques de corruption ou d'incompétence des juges) ${ }^{7}$.

Nous examinons ici un aspect central de cette régulation conjointe, la relation entre partage des coûts du contrôle et cooptation des juges, en nous appuyant sur une étude de cas, celle du Tribunal de commerce de Paris. Le tribunal de commerce est une juridiction - comme les conseils de prud'hommes pour les conflits du travail construite à partir d'un espace social localisé. Depuis le XVI ${ }^{\mathrm{e}}$ siècle, c'est elle qui a reçu de l'État le mandat de régler les conflits entre les commerçants. Elle représente une forme originale de partage des coûts du contrôle car les juges consulaires sont des hommes et des femmes d'affaires bénévoles : l'État n'a donc pas ici à payer de juges fonctionnaires, le coût salarial de la justice étant reporté sur les juges euxmêmes lorsqu'ils ne sont pas (ou plus) salariés, ou sur les entreprises dont ils sont les salariés. Dans cette solution institutionnelle fondée sur l'existence d'institutions intermédiaires (de statut public mais dont les membres sont issus du monde des affaires), les coûts du contrôle sont donc partagés par l'État, les entreprises et les juges individuels.

Notre étude de cette forme d'encastrement institutionnel s'appuie plus spécifiquement, et par conséquent de manière plus

7. On peut se demander ce que ce compromis devient aujourd'hui alors que les transactions commerciales sont de plus en plus internationales, mettant en contact des traditions juridiques diverses. D'après les spécialistes (Berger, 1999 ; Dezalay et Garth, 1996 ; Swedberg, 2003 ; Volckart et Mangels, 1999 ; Calliess, 2002) une nouvelle lex mercatoria, un droit des affaires facilitant l'activité des grandes entreprises multinationales émergerait en Occident depuis les années 1960. La globalisation économique et l'intégration européenne posent de manière encore, plus précise la question des formes que devraient prendre à l'avenir les relations entre l'État, le monde des affaires et les institutions chargées de sa régulation (Djelic et Quack, 2002). 
limitée, sur l'examen du recrutement des juges consulaires à Paris. Le recrutement de Paris est différent de celui des autres tribunaux de commerce français, notamment parce que les juges consulaires parisiens sont, on le verra, plus diplômés, plus souvent cadres supérieurs et plus souvent issus du monde de la finance que leurs collègues des autres juridictions commerciales françaises. Reste que le Tribunal de commerce de Paris est souvent considéré - par les juges eux-mêmes, par les syndicats patronaux et souvent par des représentants de la Chancellerie - comme le modèle du genre, un modèle vers lequel les tentatives de réforme devraient faire converger les autres tribunaux de commerce. L'examen du recrutement des juges consulaires à Paris a donc une portée plus générale que ne le laissent supposer les spécificités de la juridiction parisienne.

\section{Une institution de régulation conjointe : le Tribunal de commerce français}

Que font les juges du commerce? Leur travail est complexe, fortement défini et encadré par la procédure, mais aussi marqué par les conflits de normes et par leur propre pouvoir souverain d'appréciation. Il n'est pas concevable, dans le cadre d'un court article, de saisir toute la complexité de leur travail, de leurs compétences et de leurs expériences. On peut, en résumé, présenter quelques généralités sur leurs tâches.

En France, il n'y a pas de tribunal unique pour tous les litiges privés, mais des tribunaux de différentes natures, localisés dans différentes villes avec un ressort géographique défini. Les tribunaux de commerce sont des juridictions spécialisées qui ont une compétence spécifique, celle de trancher les litiges économiques et commerciaux. La compétence du tribunal de commerce est définie par le Nouveau Code de procédure civile, le Code de l'organisation judiciaire et le Code de commerce. Les litiges qui relèvent de cette compétence sont, par exemple, les litiges entre commerçants ${ }^{8}$, L 121-1), les contestations relatives aux actes de commerce entre

8. Les principales catégories de professions commerciales sont (art. L 110-1 et L 110-2 Code du commerce) le négoce (achat pour revendre), l'industrie (entreprises de manufacture à l'exception de l'artisanat), le transport, les services (intermédiaires de commerce, y compris l'hôtellerie, les assurances, l'administration de biens, les sociétés immobilières, les agences de voyages, etc.), la banque et les établissements de crédit, les spectacles, les mines, l'activité navale. 
toute personne (art. L 411-4 $3^{\circ}$ COJ), aux sociétés commerciales (art. L 411-4 $2^{\circ} \mathrm{COJ}$ ), les procédures relatives aux défaillances d'entreprises commerciales ou artisanales (Code de commerce, Livre sixième), les litiges entre commerçants et non-commerçants.

Les articles 631 et suivants du Code de commerce déterminent les litiges qui doivent être portés devant le tribunal de commerce, à moins que les parties ne conviennent par avance - au moyen d'une clause compromissoire - de soumettre leurs litiges à des arbitres. Très généralement, les principales sortes de contestation entrant dans la compétence du tribunal de commerce sont relatives aux engagements et transactions entre commerçants, aux actes de commerce entre toutes personnes et des contestations entre associés pour raison d'une société de commerce. De plus, les tribunaux de commerce sont compétents en matière de faillites, ou "procédures collectives ", liquidation ou redressement (par cession ou continuation), et en matière de prévention des difficultés des entreprises. Certaines restrictions à la compétence des tribunaux de commerce portent sur les litiges relatifs aux accidents du travail, aux baux commerciaux, aux marques de fabrique et à certains brevets.

Pour résoudre ces conflits entre acteurs économiques, le juge du commerce s'appuie, entre autres, sur le droit des affaires. Ce droit repose sur une théorie générale des obligations. Les liens juridiques existant entre les parties peuvent découler d'obligations contractuelles ou extra-contractuelles (délits et quasi-délits, abus de droit, quasicontrats (art. 1372 Code civil)). En matière commerciale, le contrat se forme par accord des parties sur ses éléments essentiels. Il n'est pas nécessaire qu'il y ait un acte écrit, sauf dans certains cas où la loi l'impose (par exemple cautionnement, transaction, assurance, cession de fonds de commerce, etc.). L'article 1108 du Code civil énumère les conditions essentielles pour la validité du contrat : consentement de la personne qui s'oblige ; sa capacité de contracter ; un objet certain ; une cause licite dans l'obligation. Ainsi le juge peut être amené à évaluer la capacité de certaines personnes à contracter, le caractère déterminé, possible et licite de l'objet de l'obligation. Entre commerçants, la preuve du consentement est libre et peut être établie par tous moyens. En cas de litige, le juge ne s'arrête pas au sens littéral des termes de la convention, mais doit rechercher quelle a été la commune intention des parties (art. 1156 Code civil). Il doit interpréter les clauses ambiguës, douteuses ou non exprimées en respectant un certain nombre de règles (art. 1157 à 1164 Code civil) : par exemple, il doit suppléer les clauses d'usage, même si elles ne sont pas exprimées ; dans 
le doute, une convention doit s'interpréter en faveur du débiteur de l'obligation dont l'exécution est demandée. Le juge est amené, par exemple, à se prononcer sur l'exécution des contrats (exécution forcée ; nullité, résolution, résiliation, caducité ; suspension de l'exécution; responsabilité et dommages et intérêts en cas de nonexécution ou de mauvaise exécution créant un préjudice à l'une des partie ; fixation d'intérêts moratoires ; application de clause pénale ou modification du montant de cette clause ; octroi de délais), sur la solidarité qui doit exister entre plusieurs personnes répondant d'une même obligation vis-à-vis d'un même contractant, sur les obligations extra-contractuelles, sur l'extinction des obligations et des créances (paiement-subrogation, compensation, novation).

En résumé, le juge du commerce entre ainsi dans la variété et la complexité des contrats de tous genres et des obligations qui régissent la vie quotidienne des acteurs de l'économie. Il statue aussi en matière d'opérations financières, qui sont des actes commerciaux s'ils sont destinés à régler une opération commerciale. Il statue enfin en matière de faillites, ou "procédures collectives", avec tous les enjeux que ces procédures représentent pour l'emploi. La fonction des juges du commerce est donc de résoudre les conflits entre acteurs économiques, surtout les entreprises, et d'exercer une certaine discipline sur l'entrée dans les marchés, sur le fonctionnement des marchés et sur une grande partie des échanges économiques.

Les solutions institutionnelles connues pour régler les conflits entre commerçants peuvent différer selon les pays, voire au sein d'un même pays. La solution institutionnelle choisie par la France depuis 1563 pour résoudre ce genre de problèmes est une juridiction consulaire, le tribunal de commerce. L'État partage donc depuis bien longtemps son propre pouvoir judiciaire avec la communauté d'affaires locale. Cette institution assure le partage des coûts du contrôle entre l'État, les entreprises ou leurs représentants (syndicats patronaux) et la société civile (représentée par les juges individuels élus). Le rôle de cette juridiction spéciale est de résoudre les conflits entre acteurs économiques, surtout les entreprises (mais aussi parfois entre entreprises et particuliers) et d'exercer une certaine discipline sur l'entrée dans les marchés et les échanges économiques. Les tribunaux de commerce sont compétents en matière de contentieux et de faillites (ou "procédures collectives ", redressement ou liquidation judiciaires).

Dans ce tribunal, chaque juge agit comme juge individuel sans mandat spécifique d'un secteur de l'économie. Les juges ne sont pas 
rémunérés et ils sont élus pour un mandat de deux ou de quatre années (pour une durée totale maximale de quatorze ans) par des "délégués consulaires " eux-mêmes élus par l'ensemble des commerçants ; peuvent aussi voter les juges déjà en place et les membres de la chambre de commerce locale, si elle existe. Cela revient à un scrutin à deux degrés partant des entreprises. Ces liens étroits entre chambres de commerce et tribunaux de commerce sont renforcés à Paris par l'existence d'une structure de sélection préalable des candidats, le CIEC (Comité intersyndical des élections consulaires), qui est contrôlée par la Chambre de commerce et qui, en pratique, "fait l'élection".

Cette solution institutionnelle au problème de la résolution des conflits commerciaux en France, on l'a dit, a une longue histoire. Depuis près de cinq siècles, les commerçants contribuent ainsi à l'accomplissement d'une fonction qui relève de l'appareil d'État. Or, comme d'autres institutions situées à la frontière de l'administration et du monde de l'économie et remplissant des fonctions de régulation, les tribunaux de commerce se caractérisent par une grande discrétion, qui explique peut-être en partie leur méconnaissance par les sciences sociales. En outre, ces institutions n'entrent guère dans les schémas quelque peu caricaturaux sur l' "exception française ». On défend ou on stigmatise ainsi (souvent sous le terme générique de "jacobinisme ") la centralisation, l'hégémonie de l'État et, parallèlement, la faiblesse du syndicalisme et du mouvement associatif. D'où la fiction d'un face-à-face entre étatisme et capitalisme sauvage, qui daterait, suivant les auteurs, de la Révolution ou de l'État absolutiste. Or de récents travaux de sociologues et d'historiens ont souligné que la réalité n'était pas si simple (Hirsch, 1991 ; Chatriot et Lemercier, 2002; Lazega et Mounier, 2002, 2003; Lemercier, 2003). Le poids d'une idéologie refusant l'existence d'intérêts particuliers est indéniable; les barrières mises, pendant près d'un siècle après 1791 , à la création de toute forme de corporation ou de syndicat en témoignent. Mais en réalité, de nombreuses institutions intermédiaires ont prospéré en France, y compris depuis la Révolution. Elles ont permis, à l'échelle locale ou nationale, des formes de régulation de l'économie dans lesquelles des hommes eux-mêmes issus du commerce et de l'industrie étaient très actifs, mais qui incluaient aussi une certaine sanction de l'État (elles n'avaient pas seulement lieu entre pairs).

Le cas des tribunaux de commerce est, dans ce cadre, particulièrement intéressant parce qu'ils représentent une des seules institu- 
tions qui a traversé presque inchangée la Révolution de 1789, tout en ayant été régulièrement remise en cause de façon fondamentale. Ils sont le parfait exemple d'une institution intermédiaire dont l'existence est difficile à justifier en théorie (juridique et politique), mais dont la nécessité s'est toujours imposée dans la pratique, à la fois en vertu d'une demande des intéressés et de l'État. Créés à un moment où la montée de l'absolutisme s'accommodait très bien de l'existence de privilèges pour certains groupes sociaux (que l'État pouvait ainsi à la fois contenter et contrôler), les tribunaux de commerce sont en effet parvenus à survivre aux périodes de jacobinisme le plus exacerbé : comprendre cette persistance d'une juridiction d'exception nécessite de s'interroger sur l'intérêt qu'ont pu y trouver différents groupes sociaux aussi bien que l'administration.

Généralement, le monde des affaires a certaines attentes vis-à-vis des juridictions commerciales, et ce depuis leurs origines : la rapidité et la fermeté, peu d'appels, une séparation nette entre le patronage personnel et la prise de décisions judiciaires, ainsi que le plus de neutralité possible. De plus, dans le cas des tribunaux consulaires, des secteurs entiers du monde des affaires, représentés par leurs syndicats patronaux, ont intérêt à être représentés au tribunal, notamment pour défendre les coutumes liées à leur activité ou à leur profession (Carruthers et Halliday, 1998 : 488) - ce qui peut entrer en conflit avec la conception d'un juge totalement neutre. Ainsi, du point de vue de certains secteurs de l'économie, les juges consulaires sont plus que de simples juges. Ils seraient des entrepreneurs judiciaires (McIntosh et Cates, 1997) qui représentent la sensibilité du syndicat patronal qui les a initialement aidés à siéger au tribunal en parrainant leur candidature ${ }^{9}$. En pratique, il est donc difficile de dissocier totalement représentativité et compétences: pour être

9. Les juges eux-mêmes ne partagent pas cet avis, en tout cas pour eux-mêmes, même s'ils le pensent au sujet d'autres juges. Lors de nos entretiens, ils affirment qu'une fois nommés, ils sont indépendants et impartiaux, qu'ils n'ont aucun mandat de leur secteur d'origine. Selon eux, rappeler les usages d'une profession ne signifie pas nécessairement représenter des intérêts corporatistes. Les médias ont souvent soupçonné que la cooptation dans les nominations avait pour résultat l'élection de juges qui ne parviendraient pas, par la suite, à se distancier de leur milieu d'origine, par exemple du secteur qui avait parrainé leur candidature, et donc d'intérêts collectifs corporatistes. Au risque que le juge soit le représentant d'un syndicat ou d'un secteur s'ajoute le risque de partialité en faveur de proches (qui est normalement contrôlé par le fait, pour le juge, de se récuser). Les parties n'ont souvent qu'une confiance limitée en l'impartialité des décisions du tribunal, particulièrement dans les tribunaux de commerce des petites villes. Elles craignent par exemple que les juges de ces petits tribunaux puissent exercer un certain contrôle judiciaire sur leurs propres concurrents (Black, 1976). 
assuré de comprendre des juges connaisseurs de tous les secteurs économiques, le tribunal doit être d'une certaine façon représentatif, ce qui pose le problème d'une manipulation éventuelle des juges par leur syndicat. Le système n'en représente pas moins un régime typique de régulation conjointe où les institutions, le monde des affaires et les particuliers acceptent de partager ensemble les coûts du contrôle.

Le tribunal de commerce français a donc des caractéristiques spécifiques qui donnent une visibilité accrue à certains aspects du lien entre les mécanismes juridiques (exogènes) et sociaux (endogènes) de la régulation dans le domaine du commerce. L'État ne sanctionne pas tout seul. Il requiert la participation et les investissements d'intervenants - tant des particuliers que des entreprises - qui sont prêts à faire leur part et à assumer une partie des coûts du contrôle. De fait, les juges élus accomplissent ce qui est habituellement considéré comme une fonction régalienne de l'État. Ce cas assez particulier de régulation conjointe se rapproche de la «corégulation " (Grabosky et Braithwaite, 1986, 83), entendue comme une autorégulation par des associations industrielles qui sont reconnues par l'Etat.

Nous proposons dans la suite de cet article d'examiner le lien entre cette forme de régulation conjointe ou de partage des coûts du contrôle et les mécanismes de cooptation des juges consulaires. Nous utiliserons pour cela nos observations du Tribunal de commerce de Paris. Ce tribunal est différent des autres tribunaux de commerce français par sa taille plus importante, par le nombre de ses chambres spécialisées (par exemple, droit des sociétés, droit international, droit communautaire, multimédia et nouvelles technologies, fonds de commerce, concurrence déloyale et contrefaçon, etc.), ainsi que par le nombre d'affaires traitées. À lui seul, le Tribunal de commerce de Paris traite 10 à $12 \%$ de l'ensemble du contentieux commercial de premier ressort traité en France (arbitrage non compris). Il est différent également par la diversité des secteurs de l'économie représentés, par ses juges, par l'importance de la clientèle, puisque la demande et la défense des sociétés se fait à leur siège social.

L'examen des procédures de sélection et des parcours des juges du Tribunal de commerce de Paris montre non seulement que cette cooptation est à la fois formelle et informelle, mais qu'elle se présente en pratique de manières différentes selon les diverses caractéristiques des juges. Elle suit des sentiers relationnels différents et 
s'accompagne aussi de légitimations différentes selon que le juge est, par exemple, un jeune chef d'entreprise ou un cadre supérieur retraité de la banque. L'étude de ces modalités de cooptation permet ainsi d'associer des modes d'encastrement de l'économie (institutionnel et relationnel) qui fonctionnent de manière conjointe mais qui sont rarement examinés ensemble. Nous interprétons enfin l'existence de cette variété de modes de cooptation comme un facteur explicatif de l'étonnante permanence de cette forme de partage des coûts du contrôle social du monde des affaires. Cette variété reflète en effet un effort particulier de la part des juges consulaires pour maintenir leur indépendance vis-à-vis de l'État tout en assurant la répartition des coûts du contrôle entre eux-mêmes, l'État et le monde des affaires.

\section{Les juges du Tribunal de commerce de Paris}

Si l'État, les entreprises et les syndicats patronaux ont toujours eu un intérêt clair au partage des coûts du contrôle, il n'en va pas tout à fait de même pour les juges individuels, dont le recrutement a toujours été assez difficile. Pour eux, la question se pose en termes plus complexes. Les motivations des juges du commerce sont nombreuses ${ }^{10}$, mais elles peuvent se résumer à l'acquisition ou au maintien d'un statut social. La notion de statut social est importante pour comprendre l'investissement bénévole des juges contemporains au sein des tribunaux de commerce ${ }^{11}$. Les motivations pour devenir juge consulaire sont souvent liées, au capital symbolique que cette fonction confere. Entre «les deux rêves du commerce» (Hirsch, 1991), valorisant l'aventure entrepreneuriale mais soucieux aussi d'ordre institutionnel, la construction actuelle du sens de cet engagement passe souvent par les honneurs, le plaisir (intellectuel, par exemple, mais aussi lié à

10. Nous ne parlons ici que des motivations dont les juges eux-mêmes font part au cours des entretiens.

11. Le bénévolat consulaire est parfois envisagé comme un moyen de gérer une " mise au placard" du juge dans son entreprise ou comme une manière de préparer un départ vers une retraite active. Dans certains cas, la fonction de juge consulaire et bénévole peut aussi mener, après la judicature, à des fonctions plus lucratives, comme celles d'arbitre dans les tribunaux d'arbitrage. Dans le cas des juges les plus jeunes du Tribunal, le statut permet l'accumulation d'un capital relationnel et l'acquisition d'une expérience importante dans le monde des affaires - avantages explicitement mis en valeur par les appels à candidature officiels du CIEC. Pour davantage d'informations sur les motivations des juges consulaires, voir Lazega, Mounier et al. (2003). 
l'exercice d'un réel pouvoir) et la valorisation sociale attachés à cette fonction. Avec cette accession, les individus concernés deviennent des personnes de référence au sein de leur milieu professionnel : élus par leurs pairs, ils jugent leurs pairs. Devenir magistrat consulaire, surtout au Tribunal de commerce de Paris, est d'abord ressenti comme une marque de reconnaissance sociale.

La conquête et le maintien de cette forme de statut sont d'autant plus difficiles pour les hommes et femmes d'affaires qu'il existe un clivage fort, dans la société française, entre gens du public et gens du privé (Singly et Thélot, 1988). Les corps intermédiaires qui existent à la frontière de ces deux mondes - dont les institutions économiques comme les chambres de commerce et les tribunaux de commerce - offrent aux hommes et femmes d'affaires l'occasion d'acquérir une forme de statut social et de distinction qui n'appartient pas au monde des affaires. L'acquisition de ce statut et de cette distinction va de pair, au moment de l'entrée comme juge au Tribunal de commerce de Paris, avec une sélection sociale. Beaucoup de juges des grands tribunaux de commerce affirment en effet posséder une certaine supériorité sociale qu'ils considèrent comme une garantie d'intégrité, essentielle à la protection de leur fonction contre l'intéressement et la corruption. Par exemple, alors que le Tribunal de commerce de Paris juge des artisans, les artisans ne sont pas juges dans ce tribunal. Comme dans les professions libérales traditionnelles, la quantité de travail difficile et non rémunéré est considérée parfois comme l'équivalent d'un rite de passage sans fin et de subordination à un idéal ${ }^{12}$. Rares sont ceux qui, sans d'importantes ressources personnelles et organisationnelles, peuvent donc se permettre de faire partie de ce que l'un des juges interviewés appelle «le club le plus cher de Paris".

Nous proposons dans cet article de montrer que cette stratégie d'acquisition ou de maintien du statut social des juges consulaires passe par une sélection sociale, sélection inséparable, nous semblet-il, du partage des coûts du contrôle social du monde des affaires. Cette sélection repose, entre autres, sur un mode de reproduction

12. Pour un aperçu de la discipline sociale que le Tribunal impose à ses juges, voir les guides d'accueil des juges consulaires nouvellement élus, intitulés Us et contumes et Le juge consulaire, en particulier "Comportement des juges entre eux - rapports hiérarchiques" (Le juge consulaire, Section V, p. 13) et "Comportement des juges dans l'exercice de sa fonction juridictionnelle ", p. 15-18. Les juges sont divisés sur l'importance de ces traditions. Les uns se disent heureux de les voir disparaitre progressivement avec les contraintes qu'elles imposent. D'autres se disent soucieux de l'avenir d'une communauté des juges qui ne s'appuierait pas sur ce "ciment puissant de cohésion du Tribunal" (Le juge consulaire, p. 13). 
basé sur la cooptation des nouveaux juges par les juges déjà en place. La longévité étonnante de cette institution s'expliquerait ainsi non seulement par les "économies» qu'elle représente pour l'État, ou encore par son pragmatisme juridique adapté aux exigences du monde des affaires (Kirat, 2001 ; Llewellyn, 1962), mais aussi parce qu'elle met en œuvre des mécanismes contrôlant l'accès à une forme de statut social particulière et valorisée dans le milieu des hommes et des femmes d'affaires. Notre démonstration part de l'identification des profils des juges du Tribunal de commerce de Paris et de la reconstitution de quelques étapes importantes de leurs trajectoires pour accéder au tribunal. Cette démonstration est fondée sur une étude empirique. Nous avons mené en 2002 des entretiens approfondis avec 182 juges du Tribunal de commerce de Paris, incluant les 156 juges en exercice à cette date, ainsi que 26 juges qui ont quitté le tribunal et ne sont plus en exercice depuis 2000.

Dans les pages qui suivent, il s'agit d'examiner certaines caractéristiques des personnes qui se lancent dans ce type d'activité à la fois bénévole et socialement complexe, d'explorer les raisons qu'elles avancent de cette participation ainsi que les modalités de leur entrée au tribunal. Pour vérifier l'hypothèse de la cooptation, nous examinons d'abord les caractéristiques sociodémographiques des juges interviewés et nous en proposons une typologie. Nous envisageons ensuite les parcours qui ont amené les juges à être candidats à cette fonction. En effet, il existe des critères de sélection complexes qui vont au-delà des conditions formelles et réglementées. Enfin, nous nous interrogeons sur la manière dont ils ont pris la décision d'intégrer le Tribunal de commerce de Paris.

\section{Les secteurs d'activité dominants}

Selon la justification du système de régulation conjointe, la sélection des juges devrait produire une représentation très diversifiée des secteurs de l'économie, particulièrement dans les grands tribunaux de commerce comme celui de Paris. Au moment de notre étude, les secteurs "représentés " par les juges (dans lesquels ils travaillaient ou avaient travaillé) étaient en effet très diversifiés. Cette diversité garantit que, quel que soit le degré de spécialisation d'une affaire, les renseignements nécessaires (caractéristiques techniques, usages professionnels, etc.) pour produire un jugement sont accessibles au tribunal par l'intermédiaire des juges issus du secteur concerné. En théorie, tous les syndicats patronaux peuvent présen- 
ter des candidats aux élections de juges consulaires sur une base annuelle afin de combler les postes vacants, résultant d'un turnover d'environ $10 \%$ du personnel du tribunal. L'analyse montre que, dans les faits, certains le font plus systématiquement que d'autres. Par exemple, le secteur de "l'intermédiation financière " (banque, produits financiers, etc.) présente chaque année plusieurs candidats à ces élections. Un peu moins d'un tiers (28\%) des juges du Tribunal de commerce de Paris sont issus de ce secteur ${ }^{13}$, suivis de loin par les services aux entreprises $(10 \%)$ et le BTP.

Avec 44 juges consulaires qui en proviennent, le secteur de la banque-finance est clairement sur-représenté au Tribunal de commerce de Paris, en termes absolus et relatifs. En effet, ce secteur représente $3 \%$ seulement de la population active en France ${ }^{14}$ et $5,1 \%$ à Paris, où les industries de service sont sur-représentées par comparaison avec la province ${ }^{15}$. En termes de valeur ajoutée à l'économie par branche (sur la base de 1995), la part du secteur des services financiers dans la valeur ajoutée totale à l'économie française était de 5,3\% ${ }^{16}$. Traditionnellement, cette industrie est un secteur très litigieux (Cheit et Gersen, 2000 ; Guéroult et al., 1993) ${ }^{17}$ : contentieux contractuels et recouvrements de créances dominent l'activité des tribunaux de commerce et une part appréciable de ces litiges concerne, pour des raisons évidentes, aussi bien le secteur financier que le Trésor public ou, dans le cas particulier des procédures collectives, des organismes comme l'Urssaf et d'autres caisses de retraites.

Ce surinvestissement de la part du secteur financier dans le contrôle des litiges commerciaux et des faillites n'est pas nouveau ${ }^{18}$.

13. Notre étude montre que 21 d'entre eux ont été parrainés par l'Association française des banques et 5 par l'Association française des sociétés financières. Parmi les institutions financières qui employaient des juges actifs (au Tribunal de commerce de Paris seulement), la BNP-Paribas avait fourni 7 juges, Suez 4, la Société générale 4, le Crédit lyonnais 4 et le Crédit commercial de France 4.

14. Enquête Emploi 2000, Institut national de la statistique et des études économiques, version CD-Rom.

15. Institut national de la statistique et des études économiques, mensuel $\mathrm{n}^{\circ} 202$, octobre 2001, Ille-de-France à la page : "Gros plan sur l'emploi francilien en 1999 ".

16. INSEE, Comptabilité nationale, 2001 (www.insee/fr/indicateur/cnat_annu/ tableaux/t_1201_25_4.htm).

En 1998, il y avait 1237 établissements de crédit en France, comptant 25428 caissiers et 714730 employés.

17. Certains juges du Tribunal de commerce de Paris pensent que le fait de travailler dans la banque prédispose à devenir juge du commerce.

18. En réalité, cette forte présence au Tribunal s'est manifestée depuis la Révolution française (Lemercier, 2003). Auparavant, d'une part les attributions du Tribunal de commerce étaient moins fermement définies, en particulier en matière de faillites; d'autre part, les banquiers étaient en général exclus des corporations de marchands qui contrôlaient le Tribunal. 
Mais au-delà de ce cas précis, malgré les efforts du monde des affaires français pour diversifier l'origine des juges grâce à une procédure électorale complexe, seuls quelques secteurs s'organisent réellement pour assurer cette présence. Pourquoi les autres ne le font-ils pas? Les juges du Tribunal de commerce de Paris avancent plusieurs hypothèses à cet égard. Les grandes entreprises utiliseraient davantage l'arbitrage (dont les affaires ne sont pas publiques) et se désintéresseraient progressivement des tribunaux de commerce. Les syndicats moins importants n'auraient pas les ressources suffisantes à consacrer à la promotion des candidats aux postes de juges consulaires. Les ressources disponibles ne sont pas les mêmes dans la banque et dans les secteurs moins bien structurés, la petite distribution, par exemple. Certains syndicats n'en verraient même pas l'intérêt.

Au-delà de ces considérations d'ordre général, il est intéressant de constater que les divers secteurs d'activité économique ne fournissent pas le même "type " de juge au tribunal de commerce de Paris du point de vue de la fonction qu'ils exercent ou avaient exercée dans l'entreprise ${ }^{19}$. Nous distinguons quatre catégories de fonctions. Le fait d'occuper ou d'avoir occupé des fonctions de "juriste " au sein de l'entreprise est une caractéristique souvent mise en avant dans les entretiens comme ayant joué un rôle important dans la décision de devenir juge consulaire, étant donné la «double compétence ", juridique et économique, qui fait la particularité du tribunal. Cette catégorie réunit $15 \%$ des juges interviewés (soit un effectif de 24 juges). Nous avons ensuite identifié une catégorie de "chefs de petites et moyennes entreprises". Par convention, nous avons choisi de classer ici tous les juges dont les discours indiquent qu'ils sont ou étaient eux-mêmes chefs d'entreprises de moins de 500 salariés et qui n'ont ou n'avaient donc pas des fonctions de «juristes». Un tiers des juges se trouvent dans cette catégorie (soit un effectif de 53 juges). Pour les juges issus des entreprises de plus grande taille, nous avons identifié ceux qui sont ou ont été hauts cadres (managers), c'est-à-dire qui n'ont vraisemblablement pas de participation au capital de l'entreprise. Ils constituent la catégorie la plus nombreuse, avec $42 \%$ du total (soit un effectif de 66 juges). Enfin, la quatrième catégorie est constituée par les juges qui indi-

19. Les informations concernant cette fonction ont été codées sur la base des entretiens réalisés avec les juges. En effet, les catégories officielles concernant les fonctions n'apportent pas d'informations particulièrement intéressantes parce que la sélection préalable des candidats exige qu'ils aient eu des responsabilités de direction et/ou qu'ils soient dirigeants d'entreprises. 
quent avoir une participation au capital de l'entreprise dans laquelle ils travaillent $\left(\mathrm{PDG}^{20}\right)$. Ils ne représentent que $8 \%$ du total des juges présents dans l'étude (soit un effectif de 13 juges) ${ }^{21}$.

On peut donc dire que le Tribunal était composé, en 2002, par une majorité de cadres supérieurs, ce qui aura une importance du point de vue des processus de cooptation décrits plus bas.

\section{Typologie des juges}

Cette diversité du Tribunal concerne non seulement le secteur d'activité et la dernière fonction occupée dans l'entreprise, mais aussi d'autres caractéristiques comme le sexe, l'âge, l'ancienneté au tribunal ou la formation. Nous présentons ici les "classes" issues d'une analyse factorielle de quelques-unes de ces caractéristiques $^{22}$. Il s'agit donc d'une typologie inductive des juges du tribunal ${ }^{23}$.

Une première catégorie de juges regroupe des hommes actifs mais en fin de carrière. Cette classe réunit $51 \%$ des juges, soit

20. Par commodité, nous appelons PDG les PDG qui se présentent comme PDG participant au capital de «leur" entreprise.

21. Les relations entre type de juge, secteur et fonction du juge dans son entreprise sont complexes. Plus de la moitié des juges "juristes " du Tribunal de commerce de Paris sont issus des secteurs liés de la banque $(37 \%)$ et des assurances et finances $(21 \%)$. Les autres secteurs apportent peu de juristes. Les secteurs de l'informatique et des télécommunications, ceux de l'immobilier et des industries alimentaire et textile n'en apportent aucun. La situation est différente pour les "chefs de petites et moyennes entreprises". Premièrement, tous les secteurs d'activité sauf l'industrie chimique y sont représentés. Deuxièmement, le secteur qui fournit le plus de chefs d'entreprise au Tribunal de commerce de Paris est celui des services aux entreprises (17\%), le deuxième secteur le plus représenté au tribunal. En ce qui concerne les «PDG", plus de deux tiers d'entre eux provient de trois groupes de secteurs : le BTP, ciment et construction électrique (31\%), les services aux entreprises $(23 \%)$ et les transports $(15 \%)$. Ils sont en revanche quasi absents du groupe de l'informatique et télécommunications, des assurances et auxiliaires financiers, de l'édition, du cinéma et de la culture, des industries alimentaire et textile et du commerce au détail. Cette absence est sûrement un effet des caractéristiques des entreprises de plusieurs de ces secteurs qui, comme le commerce de détail, entrent surtout dans la catégorie des PME. Le plus grand nombre de "managers " provient de la banque $(42 \%)$, suivi de loin par les juges assurant cette fonction au sein de l'industrie chimique $(17 \%)$.

22. Les variables "actives" de l'analyse factorielle sont les suivantes: l'année d'entrée au tribunal, avoir ou non été élève de l'École polytechnique, avoir ou non été élève d'HEC, avoir ou non réalisé des études de droit, la fonction occupée dans l'entreprise, être en activité ou à la retraite, le secteur économique d'activité, l'âge et le sexe.

23. Les groupes se différencient fortement et aucun juge n'appartient à deux classes en même temps. Nous avons choisi de retenir une partition automatique en trois classes. Les résultats complets de la classification automatique sont présentés dans Lazega, Mounier et al. (2003). Notons que les classes ainsi définies ont un sens pour les juges euxmêmes, ce dont témoignent les propos de certains juges en fin de judicature. 
79 personnes. Le nom de cette classe est suggéré premièrement par sa composition selon le sexe, puisqu'il s'agit dans la quasi-totalité d'hommes (95\% dans cette classe contre $88 \%$ dans l'ensemble) actifs au niveau professionnel et s'approchant de l'âge de la retraite. En effet, près des trois quarts des juges en activité font partie de cette classe $(74 \%)$ et ils y sont nettement plus nombreux que parmi l'ensemble des juges interviewés (77\% contre $53 \%$ ). De même, plus souvent que dans l'ensemble, les personnes de cette classe sont âgées de 55 à 59 ans $(38 \%$ contre $22 \%)$ ou de 60 à 64 ans (40\% contre $26 \%$ ). Autrement dit, les trois quarts des juges de cette classe sont âgés de 55 à 64 ans. Toutefois, la durée de leur expérience au tribunal semble être inégale, comme le suggère le fait qu'un cinquième de cette classe est composé de juges entrés au Tribunal de commerce de Paris en 2002, alors que cette promotion ne représente qu'un dixième de l'ensemble des répondants à l'enquête (21\% contre $11 \%)$. Aucun secteur d'activité n'est surreprésenté de manière significative parmi ces hommes actifs mais en fin de carrière.

Une deuxième catégorie de juges regroupe des dirigeant(e)s de PME. Cette classe réunit $12,8 \%$ des juges interviewés, soit 20 personnes. Elle regroupe une majorité significative de «chefs de petites et moyennes entreprises " $65 \%$ contre $34 \%$ dans l'ensemble) en activité (95\% dans cette classe contre $53 \%$ dans l'ensemble). Les membres de cette classe tiennent à leur identité d'entrepreneurs ${ }^{24}$. Les femmes y sont nettement plus nombreuses que dans l'ensemble des juges ayant répondu à l'enquête (50\% contre $12 \%)$; plus de la moitié d'entre elles se retrouvent ici (52\%). À la différence de la classe précédente, un peu moins des deux tiers des juges sont en pleine activité, âgés de 36 à 44 ans (20\% ici contre $4 \%$ dans l'ensemble) et de 45 à 49 ans (40\%

24. «... vous prenez la banque untel, la société untel qui envoie les gens au Tribunal, ils leur paient tous les frais. C'est toujours une entreprise qui paie. Mais moi c'est la mienne. Donc le fauteuil d'orchestre que je me suis acheté là pour une durée de douze à quatorze ans il me coûte $50000 \mathrm{~F}$ par an. Au bout de douze ans, ça fait $600000 \mathrm{~F}$. Donc ce n'est pas gratuit. Pour ceux pour qui c'est gratuit, tant mieux pour eux. Mais ça fait une différence dans la façon d'être juge. L'appréciation de la personne qui est en face n'est pas la même. Il y a un détachement, comment dire, à un moment le justiciable s'aperçoit, en se renseignant sur le juge, qu'on est en train de dépenser de l'argent pour son problème et ça quelque part ça les touche. Les justiciables ne se comportent pas pareil, ils sont plus clairs, plus ouverts. Moi je leur dis souvent "qu'est-ce qu'il vous arrive? Je suis commerçant comme vous (...) ". Je sais qu'ils se renseignent et qu'ils ne nous parlent plus de la même manière, selon qu'on est commerçant ou mandataire social " (Un juge issu du BTP). 
contre $6 \%$ ). Un autre quart appartient au groupe d'âge de 50 à 54 ans ( $25 \%$ contre $8 \%$ au total). Enfin, une part non négligeable de cette classe est issue des secteurs de l'hôtellerie et de la restauration et du commerce de détail (35\% contre $6 \%$ au total.) À noter que $70 \%$ des juges provenant de ce groupe de secteurs est concentré dans cette classe.

Une troisième catégorie de juges regroupe des juristes et managers retraités qui représentent $36,5 \%$ du total, soit 57 juges. Elle est majoritairement composée de juges à la retraite $(96 \%$ dans cette classe contre $47 \%$ dans l'ensemble). Elle est composée pour plus des trois quarts par des «juristes" $(28 \%$ contre $15 \%$ dans l'ensemble) et des "managers" (56\% contre $42 \%)$. La majorité des juges âgés de 65 à 69 ans s'y trouve réunie (86\% de ce groupe d'âges) et la totalité de ceux âgés de 70 ans ou plus. Ensemble, les groupes d'âges mentionnés représentent les trois quarts de cette classe (respectivement $56 \%$ contre $24 \%$ et $25 \%$ contre $9 \%$ ). Les secteurs de la banque et de l'industrie chimique sont surreprésentés par rapport à l'ensemble (40\% contre $28 \%$ pour le premier, $19 \%$ contre $8 \%$ pour le second). Enfin, un bon quart de ces juges possède une formation HEC ( $28 \%$ de cette classe contre $13 \%$ au total).

En nous appuyant sur cette typologie, nous souhaitons mieux comprendre le partage des coûts du contrôle mis en œuvre par cette institution consulaire. Nous proposons en particulier d'articuler ce partage aux mécanismes de cooptation qui ont amené ces juges au Tribunal de commerce de Paris.

\section{Cooptation et partage des coûts du contrôle}

La reproduction de cette institution particulière de régulation conjointe repose sur l'élection de juges bénévoles, principaux acteurs de cette forme de régulation. On peut considérer le recrutement des juges comme un processus essentiel de la régulation conjointe. En effet, le recrutement est l'un des rares moments, en démocratie respectueuse de l'indépendance du judiciaire, où la société peut peser, en amont, sur la qualité de la justice mettant en œuvre cette forme de régulation ${ }^{25}$. Il s'agit donc ici d'examiner les

25. Voir, pour des comparaisons, Bancaud (1993) ou Frison-Roche (1999). 
mécanismes de cooptation formelle et informelle qui précèdent cette élection. Avant d'aborder les parcours d'entrée au tribunal, il convient de rappeler les normes qui encadrent, de façon plus ou moins officielle, cette entrée. En particulier, cette cooptation formelle laisse la porte ouverte au corporatisme puisque les juges doivent obtenir le soutien formel d'un syndicat professionnel pour être candidats à la fonction de juge consulaire.

\section{Cooptation formelle : le dispositif du CIEC}

On l'a vu plus haut, les juges sont élus pour un mandat de deux ou quatre années (pour une durée totale maximale de quatorze ans) par des "délégués consulaires » eux-mêmes élus par l'ensemble des commerçants; peuvent aussi voter les juges déjà en place et les membres de la chambre de commerce locale, si elle existe. À Paris, c'est à l'Assemblée générale composée de plus de 150 délégués de choisir après enquête, audition en commission et par elle-même, parmi les candidats proposés par les organisations syndicales et le Comité intersyndical des élections consulaires (CIEC), "les meilleurs et les plus représentatifs d'entre eux" - on retrouve bien l'articulation complexe entre expertise et représentativité. Les délégués consulaires sont les correspondants économiques des chambres de commerce et d'industrie pour les circonscriptions auxquelles ils appartiennent. Leurs listes électorales sont dressées par catégorie et sous-catégorie professionnelle ${ }^{26}$, ce qui revient à un scrutin à deux degrés partant des entreprises.

Le CIEC, institution dont la création remonte à 1867 et est liée à l'émergence des premières unions de syndicats patronaux, est chargé de sélectionner les candidats à la fonction de juge consulaire. Ce comité est un organisme collégial représentant l'union des syndicats professionnels patronaux de Paris (300 syndicats représentant la quasi-totalité des secteurs professionnels) et des départements voisins (Hauts-de-Seine, Seine-Saint-Denis, Val-de-Marne). La fonction du CIEC est « de rechercher, de susciter et de sélectionner pour

26. Les électeurs comprennent, entre autres, les commerçants immatriculés au registre du commerce de la circonscription de la chambre de commerce, les chefs d'entreprise inscrits au répertoire des métiers et immatriculés au même registre du commerce, etc. 
les suffrages des électeurs des candidats aux fonctions de juges des tribunaux de commerce de la circonscription (tous les ans), de membres et de délégués consulaires de la Chambre de commerce et d'industrie (tous les trois ans) ». Rappelons qu'il s'agit d'un renouvellement partiel qui a pour fonction d'atténuer les effets du changement et de favoriser l'intégration progressive de nouveaux entrants.

Parallèlement à l'accent mis sur la diversification des secteurs économiques, la recherche de compétence est une des préoccupations principales du CIEC. Elle se traduit par une vigilance importante du Comité au moment de la constitution des dossiers, où l'expérience et la réussite professionnelles sont mises en avant dans un questionnaire serré et dans un entretien avec un représentant $\mathrm{du}$ CIEC qui donne un $\mathrm{avis}^{27}$. Cette procédure très minutieuse a d'ailleurs été mise en place dès la fin du XIX ${ }^{e}$ siècle à peu près dans sa forme actuelle (Lacombrade, 2002).

Cooptation informelle: les juges consulaires, principaux " recruteurs 》 de nouveaux juges

Cependant, en amont des élections et de la cooptation formelle, les juges doivent décider individuellement de se présenter aux élections consulaires. Nous l'avons vu plus haut, outre le fait de travailler pour l'intérêt général et de rendre un service public, la conquête d'un statut social représente une motivation dominante dans les déclarations des juges interviewés. Mais comment se prend la décision de poser sa candidature et quel est le contexte de cette prise de décision? Les parcours effectifs d'entrée au tribunal, tels que nous les avons reconstitués au moyen d'entretiens, donnent une image complémentaire du recrutement des juges consulaires. Nous avons en effet recueilli des informations sur le contexte dans lequel les juges interviewés ont posé leur candidature au Tribunal, notamment au sujet des personnes qu'ils ont sollicitées pour un avis concernant cette candidature (Lazega, Mounier et al., 2003, $57-62 ; 148-150)$.

Nous avons demandé aux juges du Tribunal de commerce de Paris, à l'aide d'un tableau à remplir, s'ils avaient sollicité, à l'époque

27. Les instructions que le CIEC donne à ses enquêteurs pour apprécier les candidats reposent sur trois éléments : s'assurer que le candidat a la compétence requise, la disponibilité nécessaire, et qu'il est désintéressé. 
de leur entrée au tribunal, des opinions, avis ou conseils concernant leur candidature, et auprès de qui. Il s'agit d'une question sur la situation et sur les choix passés des juges par rapport à une activité dans laquelle ils sont encore (pour la plupart) engagés, ce qui doit conduire à une certaine prudence vis-à-vis de leurs interprétations rétrospectives. Mais la majorité des juges disent avoir demandé un avis au sujet de leur candidature à au moins une personne (122 répondants sur 156).

Si l'on s'en tient à l'analyse des caractéristiques de la première personne mentionnée pour un avis au sujet de cette candidature, on peut identifier le type de lien(s) associant le répondant aux personnes consultées selon que ces personnes sont des amis, des membres de la famille, des relations professionnelles (dans la même entreprise ou du même secteur) ou encore s'il s'agit d'un juge du tribunal ou d'un autre membre du monde judiciaire (avocat, etc.). Ces qualificatifs des relations peuvent apparaitre seuls ou en combinaison (par exemple, un ami qui fait partie du Tribunal de commerce de Paris, un membre de la famille qui est avocat, etc.). Nous avons recensé 13 qualificatifs différents utilisés par les juges interviewés pour identifier la première personne sollicitée pour un avis au sujet de leur candidature. Les relations qualifiées par une seule caractéristique (uniplexe) sont majoritaires ${ }^{28}$. Nous avons effectué une mise à plat des caractéristiques mentionnées, qu'elles soient uniplexes ou multiplexes. Ainsi, les 122 juges ayant répondu à la question ont utilisé 165 qualificatifs pour identifier le type de lien(s) qui les unit aux personnes sollicitées. Le tableau suivant résume les réponses à cette question $^{29}$.

28. $65 \%$ des relations avec la première personne citée sont uniplexes.

29. La décision d'être candidat au Tribunal de commerce de Paris est donc soumise à une diversité d'influences que l'on peut essayer de synthétiser davantage. La première catégorie de relations regroupe 66 personnes appartenant à l'institution consulaire - sans différencier les relations uniplexes et multiplexes - et 4 personnes appartenant au monde judiciaire autre que consulaire. Dans une deuxième catégorie sont réunies les relations concernant les collègues du candidat (appartenant à la même entreprise), soit 28 personnes - sans différencier les relations uniplexes et multiplexes - ainsi celles avec des personnes provenant du même secteur économique (12 personnes). La troisième catégorie est constituée par les relations amicales ( 5 personnes) et les relations familiales ( 7 personnes). 


\section{Qui consulte-t-on avant de poser sa candidature au Tribunal de commerce de Paris? (première personne citée)}

À l'univers consulaire ou judiciaire

L'avis le plus recherché avant de poser sa candidature est celui de personnes appartenant déjà à l'univers consulaire ou, dans une certaine mesure, à l'univers judiciaire plus large. Suit de près la recherche d'un avis issu de l'environnement professionnel des juges puis, largement derrière, les avis issus de leur environnement personnel ou privé. Le fait que les avis les plus nombreux au sujet d'une éventuelle candidature proviennent de juges du Tribunal de commerce de Paris ou d'un autre tribunal signifie que ce sont les membres du tribunal eux-mêmes qui jouent le rôle principal d'attraction de nouveaux juges par relations informelles aboutissant au recrutement.

Dès lors, on ne peut plus envisager la sélection des juges uniquement à partir des règles édictées par l'État et par les organismes représentant le monde économique (le CIEC et l'Assemblée générale de la Chambre de commerce). Il faut envisager aussi à partir de la dimension informelle que nous venons de mettre en évidence. Les mécanismes de reproduction formels du tribunal sont accompagnés de deux types de comportements informels : les premiers, que l'on peut appeler de "promotion ", sont le fait des participants légitimes de la régulation conjointe que sont les entreprises et syndicats patronaux. Les seconds impliquent les juges consulaires déjà en place.

Ce constat laisse cependant ouverte la question de l'articulation des deux formes, formelle et informelle, de cooptation. Pour décrire cette articulation, il convient de s'interroger davantage sur ces divers comportements de sélection informels, notamment en cherchant à déterminer s'il existe des liens entre ces comporte- 
ments et d'autres caractéristiques des juges. Examinons au préalable les circonstances d'entrée au Tribunal que les juges identifient euxmêmes.

\section{Une cooptation informelle différente selon les types de juge}

D’un côté, il existe des initiatives "personnelles ", qui émanent des futurs candidats eux-mêmes. Mais d'un autre côté, une entreprise ou un secteur d'activité économique peuvent proposer à leurs membres de se porter candidats à la fonction de juge consulaire. Un large éventail de situations apparait dans les entretiens, que nous avons regroupé en quatre types.

1 / La personne dit avoir voulu de son propre chef devenir juge et sans avoir eu à demander l'autorisation à sa hiérarchie (propre chef). Plus d'un quart des juges se trouve dans cette catégorie $(29 \%)$.

2 / Cette même volonté individuelle a dû faire l'objet d'une information à la hiérarchie de l'entreprise. Plusieurs cas de figure apparaissent : ou bien cette information est une pure exigence formelle ${ }^{30}$, ou bien le futur candidat a dû absolument obtenir l'accord de sa hiérarchie. Soit l'entreprise a été tout à fait d'accord pour que son collaborateur soit juge consulaire, soit son soutien n'a pas été automatique mais a fait l'objet d'une négociation. Cette catégorie est la plus nombreuse (78 individus, $50 \%$ du total).

3 / La personne dit n'avoir pas eu elle-même "l'idée " d'entrer au tribunal, mais avoir accepté une proposition de son syndicat professionnel (démarché par son syndicat). Dans une variante, le CIEC a approché directement la personne pour lui proposer de se présenter aux élections consulaires (soit 15 juges, $7 \%$ du total).

4 / D'autres juges (7 \%) ont posé leur candidature à la suite d'une suggestion de leur entreprise (démarché par l'entreprise) ${ }^{31}$.

Nous avons ainsi exploré la question de la cooptation informelle à travers une analyse factorielle (dont le résultat est présenté en

30. Par exemple, la personne est dans une situation de pouvoir et est libre de sa décision mais informe néanmoins son entreprise, ou bien elle ne représente plus un enjeu pour celle-ci (départ à la retraite prévu, par exemple).

31. Il y a 7 personnes ( $5 \%$ ) pour lesquelles cette information n'apparait pas dans les entretiens (sans information sur l'initiative). 
annexe). Cette analyse fait apparaitre une forte relation entre la classe à laquelle appartiennent les juges (hommes actifs mais en fin de carrière, dirigeant(e)s de PME, juristes et managers retraités), les initiatives et circonstances de candidature et les caractéristiques des personnes sollicitées pour un avis au sujet de la pertinence de la candidature ${ }^{32}$.

Le constat le plus saillant de l'analyse concerne l'opposition entre le groupe de juges identifiés plus haut comme "dirigeant(e)s de PME " et ceux qui réunissent les "hommes en fin de carrière " et les «managers et juristes retraités ». En effet, les hommes et les femmes du premier groupe déclarent le plus souvent soit avoir posé leur candidature au tribunal de leur propre initiative, soit avoir été démarchés par leur secteur ou le CIEC. Autrement dit, leur décision n'est pas majoritairement soumise à l'approbation d'une hiérarchie. Par ailleurs, ils disent majoritairement ne pas avoir discuté de la pertinence de cette candidature avec quiconque ou n'en avoir discuté qu'avec des membres de la famille ou des amis. Nous sommes donc face à un mode d'entrée au Tribunal qui semble faiblement relever de la cooptation informelle.

Rappelons que cette classe minoritaire est constituée principalement de juges qui revendiquent fortement leur identité d'entrepreneur. Ces chefs d'entreprise se réclament de ce qu'ils considèrent être les catégories fondatrices du Tribunal, c'est-à-dire les commerçants et entrepreneurs indépendants ${ }^{33}$. De ce point de vue, ces juges auraient un intérêt immédiat mais aussi traditionnel à participer aux coûts du contrôle social du monde des affaires, surtout si l'on tient compte du fait que la plus grande partie du contentieux du tribunal concerne les petites et moyennes entreprises. Ces caractéristiques expliquent que ces juges décrivent leur candidature au Tribunal comme allant de soi. Ils n'ont guère besoin de confirmer le bien-fondé de cette candidature, puisque leur participation au Tribunal leur apparait légitime, tant du point de vue de leur activité

32. Le commentaire suivant consiste en une lecture du tableau des contributions cumulées de chaque modalité à la composition des facteurs, de même que le constat de la plus ou moins grande proximité procède du tableau des coordonnées et des valeurs-test des mêmes modalités. Plus précisément, chaque facteur est "décrit " par les modalités présentant une valeur-test supérieure en valeur absolue à 2 . Notre commentaire synthétise les informations apparaissant sur les deux premiers facteurs, c'est-à-dire sur le premier plan factoriel.

33. "... on peut regretter qu'il y ait de moins en moins de commerçants, de commerçants purs, de vrais, qui représentent véritablement le juge du commerce tel qu'il était, c'est-à-dire les juges des marchés - maintenant ça n'existe plus beaucoup parce que ils n'ont plus le temps et parce que le commerce a été complètement transformé. " 
présente que du point de vue des traditions supposées de l'institution ${ }^{34}$.

En ce qui concerne les deux autres types de juges, les « hommes en fin de carrière " et les "managers et juristes retraités ", les uns et les autres déclarent fréquemment aussi avoir posé leur candidature de leur propre initiative mais, contrairement aux " dirigeant(e)s de PME ", ils ont dû soumettre cette décision à l'approbation de leur hiérarchie. Parfois, cette demande d'autorisation est purement formelle, par exemple lorsque le candidat est lui-même au sommet de cette hiérarchie. Mais la demande de confirmation de l'initiative personnelle peut aussi coïncider avec les intérêts des entreprises, qui ne voient pas d'un mauvais œil le fait d'avoir des «représentants" au Tribunal ${ }^{35}$. Dans certains cas, rares il est vrai, l'initiative personnelle de poser sa candidature se heurte à l'opposition de la hiérarchie de l'entreprise, c'est-à-dire à un refus du partage des coûts de la régulation conjointe ${ }^{36}$.

Des différences apparaissent néanmoins entre ces deux groupes. Les «hommes en fin de carrière " sollicitent fréquemment des avis concernant la pertinence de leur candidature auprès de personnes appartenant au milieu consulaire (et plus largement judiciaire). Autrement dit, s'ils ont obtenu l'accord de leur entreprise au sujet de leur candidature aux fonctions de juge, cet accord ne suffit pas pour autant pour s'y engager ${ }^{37}$. La complexité des mécanismes de cooptation au Tribunal de commerce de Paris apparait ici nettement. En effet, on comprend que la décision de s'engager dans une

34. En effet, jusqu'au $\mathrm{XX}^{\mathrm{e}}$ siècle, il fallait "faire le commerce en personne " ou l'avoir fait dans le passé, c'est-à-dire être patenté, pour être électeur et éligible. Les cadres n'avaient évidemment pas dans les entreprises la place qu'ils y tiennent aujourd'hui ; les directeurs de succursales eux-mêmes étaient encore exclus du vote dans les années 1890 (Chante-Grellet, 1897-1898). Cependant, jusqu'à la fin du XIX ${ }^{\mathrm{e}}$ siècle, les représentants du petit commerce étaient pratiquement exclus du Tribunal de commerce parisien : les juges étaient certes des indépendants, mais aussi de grands banquiers, négociants ou industriels et non des petits commerçants, le secteur alimentaire (hors des grands négociants en vins) étant particulièrement sous-représenté (Lemercier, 2003). Ce n'est qu'au $\mathrm{XX}^{\mathrm{e}}$ siècle, à la faveur du nouveau mode de scrutin et de l'émergence de nouvelles organisations syndicales, que les PME ont construit leur forte présence et, dans le même temps, le mythe de leur attachement originel à l'institution, qui apparait aujourd'hui très fortement dans les discours.

35. «... d'une part quand j'ai senti que j'étais près de la porte de sortie (...) je me suis dit qu'il y avait peut-être un intérêt à me présenter à la fonction de juge consulaire. Et d'autre part, ma direction générale était très motivée par le fait qu'il y ait quelqu'un qui soit au Tribunal de commerce."

36. "La hiérarchie compte. Si vous voulez, je n'aurais pas pu le faire sans leur accord, donc j'ai eu leur accord, mais sans enthousiasme. Je pense que je n'aurais pas pu le faire si j’avais été plus jeune."

37. "... il se trouve que j'avais des connaissances qui étaient juges consulaires à Paris ; ils m'ont reçu, ils m'ont fait visiter les lieux, ils m’ont raconté un peu comment ça se passait, donc si vous voulez je me suis renseigné... " 
activité coûteuse en temps et en argent fasse l'objet d'une certaine démarche de vérification. Il apparaît clairement que des magistrats consulaires jouent un rôle d'incitation concrète à la décision de poser candidature ${ }^{38}$. Par ailleurs, cette lourdeur de la fonction consulaire explique aussi qu'une partie de ces «hommes en fin de carrière ", mais aussi des "dirigeant(e)s de PME " aient sollicité des avis de membres de leur univers personnel ${ }^{39}$.

Quant aux «managers et juristes retraités », ils partagent avec les "hommes en fin de carrière" le fait d'avoir postulé au tribunal de leur propre initiative mais en ayant obtenu l'accord de l'entreprise. Mais ils peuvent aussi avoir été incités à se présenter aux élections consulaires par leur syndicat professionnel ou par le CIEC luimême ${ }^{40}$. Parfois, mais dans une moindre mesure, ils ont été "démarchés" par l'entreprise où ils travaillent (ou travaillaient à l'époque). Ces juges déclarent ne pas avoir eu l'initiative de la candidature, mais plutôt avoir répondu aux souhaits de l'entreprise ${ }^{41}$.

Lorsque la candidature a été suggérée par l'entreprise ou par le secteur d'activité (ou le CIEC) de manière explicite, la demande d'avis à des représentants du monde consulaire est moins fréquente. Les candidats sollicitent plutôt des opinions dans leur milieu professionnel. Autrement dit, les candidatures soutenues par les syndicats professionnels ou les entreprises suscitent moins de démarches de confirmation auprès du monde consulaire. Nous sommes ici devant le cas le plus net de participation du monde des affaires aux coûts de la régulation conjointe de ses conflits.

L'analyse qui précède permet de formuler des propositions bien fondées au sujet de la relation entre partage des coûts du contrôle et cooptation informelle des juges du Tribunal de commerce de Paris. La petite classe des " dirigeant(e)s de PME » est entrée au Tribunal de sa propre initiative, confortée par sa légitimité traditionnelle (même si cette tradition est en partie un mythe). Du fait de cette sécurité concernant la pertinence de leur participation, ces juges n'ont guère fait appel à des avis "éclairés " au moment de leur candidature

38. «... j'avais des amis qui étaient au tribunal et qui m'ont dit combien c'était passionnant..."

39. "J'ai pris une décision personnelle... familiale. On ne peut pas être juge sans en parler à son épouse au moins. Donc c'est une consultation familiale, voilà, mais personnelle."

40. "J'ai été contacté par le syndicat de la banque dont je fais partie et qui a envoyé un formulaire à tous ses adhérents pour trouver des juges consulaires. "

41. "... être juge consulaire ne faisait pas partie de mes projets personnels. On m'a demandé (l'entreprise) et j'ai eu à faire le choix d'accepter ou de refuser (...).» 
auprès de juges déjà en place ou auprès des autres acteurs institutionnels qui partagent avec l'État les coûts de la régulation conjointe : les syndicats professionnels et la Chambre de commerce d'une part, les entreprises d'autre part. Plus nombreux, les autres juges présentent une variété de situations recouvrant le gros des processus de cooptation informelle au Tribunal de commerce de Paris. Ainsi, ceux qui ont dû solliciter l'autorisation de leur hiérarchie pour pouvoir présenter leur candidature à la fonction de juge font néanmoins état d'une "entrée " informelle au Tribunal ; pour se renseigner et obtenir un avis au sujet de leur candidature, ils se tournent plus fréquemment vers des membres du monde consulaire et judiciaire. Il s'agit d'un type d'encastrement particulier donnant lieu à la forme la plus claire de cooptation de la part du Tribunal. Enfin, d'autres juges ont été "poussés » à se présenter soit par leur syndicat professionnel, soit par leur entreprise. Cet encastrement organisationnel ou institutionnel fait appel à une autre forme de confirmation, puisque les avis sollicités par ces juges sont orientés vers des personnes appartenant à leur univers professionnel.

\section{L'étonnante permanence de cette institution de régulation conjointe}

La solution institutionnelle adoptée en France il y a quatre siècles et demi pour mettre en œuvre le contrôle social du monde des affaires surprend par sa longévité. L'examen de cette solution est important, nous semble-t-il, car elle prétend réunir les admirateurs de la vertu républicaine et les partisans de l'esprit de commerce dans l'économie de marché. Déjà Montesquieu soulignait les limites de l'opposition entre la république et le commerce, entre l'intérêt général et l'intérêt individuel $^{42}$ (Manin, 2001). Cette opposition, qui s'est imposée pour-

42. "Le commerce est la chose du monde la plus utile à un État " (Montesquieu, Lois, XXI, 14). Ou encore : "Il est vrai que lorsque la démocratie est fondée sur le commerce, il peut fort bien arriver que des particuliers y aient de grandes richesses, et que les mœurs n'y soient pas corrompues. C'est que l'esprit de commerce entraîne avec soi celui de frugalité, d'économie, de modération, de travail, de sagesse, de tranquillité, d'ordre et de règle. Ainsi, tant que cet esprit subsiste, les richesses qu'il produit n'ont aucun mauvais effet " (Lois, V, 6). La même discipline serait requise dans la vertu républicaine et dans l'esprit de commerce, pourvu que les phénomènes de corruption soient rendus publics et exposés à la critique. La question des relations entre vertu et commerce dans la pensée économique et politique a été largement étudiée, pour l'Angleterre, par les historiens de l' "école de Cambridge" (Hont et Ignatieff, 1983). Des études comparables manquent encore en France, l'histoire de la pensée jacobine ou républicaine ayant souvent laissé peu de place aux aspects économiques. 
tant comme une idée dominante dans l'histoire des idées politiques, est remise en question par l'institution consulaire.

Nous interprétons l'existence de cette variété de modes de cooptation comme un facteur explicatif de l'étonnante permanence de ce partage des coûts du contrôle social du monde des affaires. Cette variété reflète en effet, de la part de ce monde très hétérogène, un effort particulier pour conserver le contrôle de lui-même (se soustraire à celui de l'Etat), malgré son hétérogénéité, tout en répartissant ces coûts entre une partie de ses membres.

Cette cooptation va de pair avec l'affirmation des intérêts corporatistes de certains secteurs de l'économie, mais aussi avec l'une des motivations principales de la plupart des juges contemporains : la conquête du statut social. Comme dans les professions libérales traditionnelles, l'entrée comme juge au Tribunal de commerce de Paris fait l'objet d'une sélection sociale. Certains associent, dans leurs propos sur la noblesse de leur tâche, cette supériorité sociale à une vertu quasi aristocratique. Comme dans les professions établies, les nouveaux membres assimilent une culture organisationnelle et une discipline pour être acceptés. Cette culture et cette discipline remplacent, en principe, celles de leurs secteurs d'origine.

L'une des difficultés de cette institution consulaire (et du partage des coûts du contrôle qu'elle représente) réside dans le recrutement des juges consulaires. Nous avons vu que plusieurs motivations guident leur approche du Tribunal de commerce. La cooptation informelle que nous avons décrite représente ainsi une condition d'existence du partage des coûts du contrôle entre État, entreprises et société civile. Cette condition d'existence est mieux mise au jour par l'analyse sociologique que ne le permettrait une approche économiste, fût-elle néo-institutionnaliste, ou juridique.

Si l'étude des modalités de recrutement des juges consulaires à Paris $^{43}$, en particulier des modalités de leur cooptation, permet d'expliquer en partie la longévité de cette institution, elle permet aussi de formuler une hypothèse plus générale. Plus l'État délègue à des représentants de la société civile locale des fonctions aussi importantes, plus la cooptation des acteurs de cette régulation conjointe devient forte et sélective, voire élitiste. Deux modes d'encastrement

43. Il faut souligner que les formes de cooptation identifiées plus haut sont sans doute assez spécifiques de Paris, où la taille des juridictions est associée à l'existence d'un "vivier" de compétences suffisamment large pour donner sens à une organisation comme le CIEC. Reste que d'autres formes de cooptation sont possibles et probables dans d'autres juridictions consulaires. 
de l'économie (institutionnel et relationnel) - qui fonctionnent de manière conjointe mais qui sont rarement examinés ensemble - sont ainsi associés dans les modalités de cooptation des juges. Plus généralement, ce fonctionnement conjoint mérite, nous semble-t-il, davantage d'intérêt de la part de la sociologie économique.

Ana Maria FALCONI $L A S M A S-C N R S$

Karima GUENFOUD CRESP-INSERM

Université de Paris XIII

Emmanuel LAZEGA Institut de sociologie, CLERSÉ-CNRS Université de Lille I et IUF

Claire LEMERCIER IHMC-CNRS

École normale supérieure

Lise MOUNIER

LASMAS-CNRS

\section{RÉFÉRENCES BIBLIOGRAPHIQUES}

Abulafia M., 1997, Making Markets, Cambridge, Mass., Harvard University Press.

Ayres I. et Braithwaite J., 1992, Responsive Regulation Transcending the Deregulation Debate, Oxford, Oxford University Press.

Bancaud A., 1993, La haute magistrature judiciaire entre politique et sacerdoce, ou le culte des vertus moyennes, Paris, LGDJ.

Berger K. P., 1999, The Creeping Codification of the Lex Mercatoria, The Hague, Kluwer Law International.

Berman H., 1983, in Law and Revolution: The Formation of the Western Legal Tradition, p. 333-356, Cambridge, Harvard University Press.

Black D., 1976, The Behavior of Law, New York, Academic Press.

Black D. 1984, "Social control as a dependent variable ", in Black D. (ed.), Toward a General Theory of Social Control, New York, Academic Press.

Buskens V., Raub W. et Snijders C. (eds.), 2003, The Governance of Relations in Markets and Organizations, Research in the Sociology of Organizations, vol. 20, Oxford, JAI/Elsevier.

Calliess G.-P., 2002, «Reflexive transnational law: The privatisation of civil law and the civilization on private law ", Zeitschrift für Rechtssoziologie, 23, p. 185-216. 
Campbell J. L. et Leon N. L., 1991, Governance of the American Economy, Cambridge England and New York, Cambridge University Press.

Carruthers B. G. et Halliday T., 1998, Rescuing Business : The Making of Corporate Bankruptcy Law in England and the United States, Oxford, Oxford University Press.

Chante-Grellet A., 1897-1898, Traité des élections, Paris, P. Dupont.

Chatriot A. et Lemercier C., 2002, "Les corps intermédiaires", in Duclert V. et Prochasson C. (dir.), Dictionnaire critique de la République, Paris, Flammarion, p. 691-698.

Cheit R. E. et Gersen J. E., 2000, "When businesses sue each other : An empirical study of state court litigation ", Law \& Social Inquiry, 25, p. 789816.

Coutant, M.-F., 1998, Les Tribaunaux de commerce, Paris, Presses Universitaires de France.

Dezalay Y. et Bryant G., 1996, Dealing in Virtue : International Commercial Arbitration and the Construction of a Transnational Legal Order, Chicago, University of Chicago Press.

Djelic M.-L. et Quack S. (eds), 2002, Globalization and Institutions, Cheltenham, Edward Elgar.

Dunworth T. et Rogers J., 1996, "Corporations in court: Big business litigation in US Federal Courts : 1971-1991 ", Law \& Social Inquiry, vol. 21, p. 497-592.

Evans P., Rueschmeyer D. et Skocpol T., 1985, Bringing the State back in, Cambridge, Cambridge University Press.

Evans P., 1995, Embedded autonomy: States and Industrial transformations, Princeton, NJ, Princeton University Press.

Fligstein N., 2001, The Architecture of Markets : An Economic Sociology of Twenty-first-Century Capitalist Societies, Princeton, Princeton University Press.

Frison-Roche M.-F., 1999, Droit, finance, autorité. Les modes de régulation juridique propres aux autorités de marchés financiers, Paris, Rapport à la mission de recherche "Droit et justice ", Ministère de la Justice.

Grabosky P. et Braithwaite J., 1986, Of Manners Gentle : Enforcement Strategies of Australian Business Regulatory Agencies, Oxford, Oxford University Press.

Granovetter M. S., 1985, «Economic action and social structure : The problem of embeddedness", American Sociological Review, 91, p. 481-510.

Greif A., 1998, "Cultural beliefs and the organization of society : A historical and theoretical reflection on collectivist and individualistic societies ", in Brinton M. et Nee V. (eds), The New Institutionalism in Socio$\log \gamma$, New York, Russell Sage Foundation.

Guéroult A., Lamotte H. et du Marais B., 1993, Les garanties et le crédit aux entreprises, Rapport du Conseil national du crédit, Paris, Éditions de la Banque de France. 
Hamilton G. et Feenstra R., 1998, "The organization of economies ", in Brinton M. et Nee V. (eds), The New Institutionalism in Sociology, New York, Russell Sage Foundation.

Hirsch J.-P., 1991, Les deux rêves du commerce. Entreprise et institution dans la région lilloise (1780-1860), Paris, Éditions de l'EHESS.

Hont I. et Ignatieff M. (eds), 1983, Wealth and Virtue: The Shaping of Political Economy in the Scottish Enlightenment, Cambridge, Cambridge University Press.

Kirat T., 2001, "L'économie et la jurisprudence : étude de plusieurs registres d'interconnexion ", Économie appliquée, 54, p. 149-179.

Lacombrade P., 2002, La Chambre de commerce de Paris et le capitalisme français (1890-1914), thèse de doctorat d'histoire, Université de Paris X.

Lazega E. et Mounier L., 2002, "interdependent entrepreneurs and the social discipline of their cooperation : structural economic sociology for a society of organizations ", in Favereau O. et Lazega E. (eds), Conventions and Structures in Economic Organization: Markets, Networks and Hierarchies, Cheltenham, UK, Edward Elgar Publishers.

Lazega E., Mounier L., 2003 a, "Interlocking Judges: On Joint (External and Self) Governance of Markets ", in Vincent Buskens, Werner Raub and Chris Snijders (eds), Research in the sociology of Organizations.

Lazega E., Mounier L. et al., 2003, Régulation conjointe et partage des compétences entre les juges du Tribunal de commerce de Paris, Rapport de recherche, Mission de recherche "Droit et justice ", juillet 2003.

Lemercier C., 2003, Un si discret pouvoir. Aux origines de la Chambre de commerce de Paris, 1803-1853, Paris, La Découverte.

Llewellyn K., 1962, Jurisprudence : Realism in Theory and Practice, Chicago, University of Chicago Press.

Macaulay S., 1986, "Private government », in Lipson L. et Wheeler S. (eds), Law and the Social Sciences, New York, Russell Sage, p. 445-518.

Manin B., 2001, "Montesquieu, la république et le commerce ", Archives européennes de sociologie, 42, p. 573-602.

McIntosh W. V. et Cates C. L., 1997, Judicial Entrepreneurship : The Role of the Judge in the Marketplace of Ideas, Westport, CT, Greenwood Press.

Milgrom P. R., North D. C. et Weingast B. R., 1990, "the role of institutions in the revival of trade: The law merchant, private judge, and the champagne fairs ", Economics and Politics, 2, p. 1-23.

Nelson R. et Winter S., 1982, An Evolutionary Theory of Economic Change, Cambridge, Mass., Harvard University Press.

North D., 1990, Institutions, Institutional Change, and Economic Performance, Cambridge, Cambridge University Press.

North D. et Thomas P., 1976, The Rise of the Western World : A New Economic History, Cambridge, Cambridge University Press.

Posner E., 2000, Law and Social Norms, Cambridge, Mass., Harvard University Press. 
Raub W. et Weesie J. (dir.), 2000, The Management of Durable Relations : Theoretical and Empirical Models for Households and Organizations, Amsterdam, Thela Publishers.

Reiss A. J. Jr., 1984, "Selecting strategies of social control over organizational life ", in Hawkins K. O. et Thomas J. M. (dir.), Enforcing Regulation, Boston, Kluwer-Nijhof.

Reynaud J.-D., 1989, Les règles du jeu, Paris, Armand Colin.

Schelling T. C., 1960, The Strategy of Conflict, Oxford, Oxford University Press.

Singly F. de et Thélot C., 1988, Gens du privé, gens du public : la grande différence, Paris, Dunod.

Stone C. D., 1975, Where the Law Ends: The Social Control of Corporate Behavior, New York, Harper \& Row.

Swedberg R., 1993, "Economics and custom ", Journal of Institutional and Theoretical Economics, vol. 149, p. 204-209.

Swedberg R., 1998, Max Weber and the Idea of Economic Sociology, Princeton, Princeton University Press.

Swedberg R., 2003, "The case for an economic sociology of law », Theory and Society, vol. 32, 1.

Vaughan D., 1983, Controlling Unlawful Organizational Behavior, Chicago, Chicago University Press.

Volckart O. et Mangels A., 1999, "Are the roots of the modern lex mercatoria really medieval ?", Southern Economic Journal, 65, 3, 427-450.

Weber M., 1988 [1889], "Zur Geschichte der Handelsgesellschaften im Mittelalter ", in Gesammelte Aufsätze zur Sozial- und Wirtschaftsgeschichte, Tübingen, J. C. B. Mohr, p. 312-443.

Weber M., 2000 [1894], Die Börse, in Theory and Society, vol. 29, p. 303337, traduit par Steven Lestition.

White H. C., 2001, Markets from Networks, Princeton, Princeton University Press.

Whitley R., 1992, Business Systems in Asia, London, Sage.

Williamson O., 1975, Markets and hierarchies, New York, Free Press.

Williamson O., 1985, The Economic Institutions of Capitalism, New York, Free Press.

Williamson O., 1996, The Mechanisms of governance, New York, Oxford University Press.

\section{Annexe}

Mode de lecture du plan factoriel : les points proches tendent à caractériser ensemble une sous-population ou groupe de personnes. Plus on s'approche du centre, plus ces caractéristiques sont partagées par l'ensemble de la population. On dit alors qu'elles ne sont pas "discriminantes ". Pour la lecture substantive, voir dans le texte. 


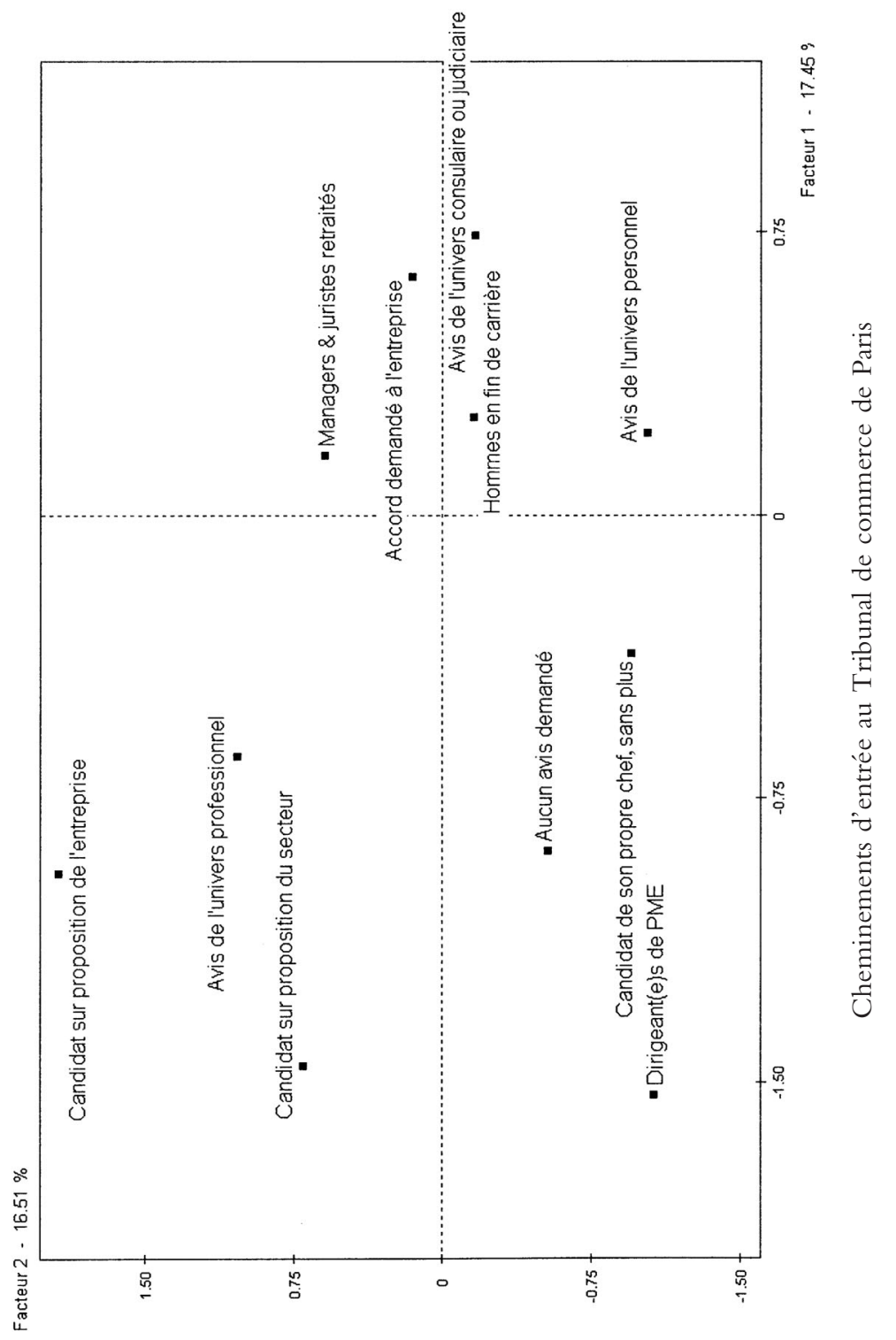

